«Faulheit» mit ihm assoziieren (das Beispiel ist nicht frei erfunden). Die Biologen und Anthropologen wären schlecht beraten, den Rassebegriff aufzugeben, weil besonders zwischen 1933 und 1945 wirklich katastrophaler Mißbrauch damit getrieben wurde.

Zusammenfassend: Es scheint mir, daß der Ansatz von $\mathrm{C}_{\mathrm{AROL}}$ ein durchaus tragfähiges Fundament für eine eigenständige Wissenschaft Geographie abgibt allerdings nur unter einer einschränkenden Bedin- gung: daß man der Geographie nicht mehr zumutet, als man es anderen Wissenschaften gegenüber tut: beispielsweise einer so ehrwürdigen Wissenschaft wie der Physik.

\section{Danksagung}

Ich danke $H$. WANNER für Literaturangaben und Diskussionsmöglichkeit und P.Schulthess für seine kritische Lektüre dieses Manuskripts.

\title{
Diskussion zum Vortrag von P. Hoyningen
}

\author{
Teilnehmerliste \\ PH Dr. PAUL HOYNINGEN, Physiker und Wissenschafts- \\ theoretiker, Zürich (Referent) \\ KI Dr. KLAUS ITTEN, Geograph, Zürich (Diskussionsleiter) \\ HB Dr. HANS R. BRUNNER, Geograph und Soziologe, Zürich \\ PB PETER BÜNZLI, Geographiestudent, Zürich \\ EE Prof. Dr. EMIL EGLI, Geograph, Zürich \\ TK THEO KELLER, Geograph, Zürich \\ RN RUEDI NÄGELI, Geograph, Bern \\ ER ERICH RENNER, Geograph, Zürich \\ CS CHRISTIAN SCHMID, Geographiestudent, Zürich \\ TS THOMAS SCHWEIZER, Geographiestudent, Zürich \\ HW HERBERT WANNER, Geograph, Zürich \\ OW Prof.Dr. OTTO WERNLI, Geograph, Zürich
}

$\mathrm{KI}$ : Wir haben beim letzten Vortrag von Dr.H.R.BRUNNER einiges über die Zürcher Landschaftsschulen erfahren. Bei diesem Referat geht es nun um einen Vertreter der jüngeren Zürcher Landschaftsschule, um HaNS CaROL. Bei dieser Gelegenheit möchte ich noch Herrn Prof. O. WERNLI vorstellen, er war ein Mitstreiter von HANS CaROL und hat in seiner Dissertation zur Diskussion um den Landschaftsbegriff beigetragen. Ich möchte die Diskussion mit einer Frage eröffnen: Darf man das Aufsteigen von feuchten Luftmassen über ein Gebirge und das Ausregnen, ich bezeichne dies als etwas Triviales, heranziehen als Beispiel für eine Korrelation?

PH: Da möchte ich auf zwei Ebenen antworten. Einerseits wird die Existenz von Korrelationen natürlich auch mit trivialen Beispielen bewiesen. Anderseits ist es forschungspsychologisch natürlich besser, wenn man interessante Korrelationen zeigt. $\mathrm{Da}$ der Kohlenstoff eines der wesentlichen Elemente beim Aufbau von Lebewesen ist, hat natürlich nicht die Molekularbiologie ins Laufen gebracht. Dazu hat man interessantere Sachen gebracht, wie die Untersuchung des Hämoglobins. Dort hat man gefunden, wie man aus der Struktur eines Moleküls die biologischen Funktionen ableiten kann, wie es das Molekül schafft, Sauerstoff zu transportieren. Das ist ein interessantes Beispiel. Interessante Korrelationen muß man bringen, damit sich die Leute auch um diese neu konstituierte Wissenschaft kümmern.

PB: Wenn Sie die Prämissen, die Sie für die Wissenschaft genannt haben, aufrechterhalten, dann ist in
Ihrem Wissenschaftsbegriff auch das organisierte Verbrechertum mit enthalten. Die Konsequenz daraus wäre, daß man diese Definition von Wissenschaft etwas enger fassen müßte, z.B. indem man den Begriff des Forschungsprogrammes mit hineinnehmen würde. Wenn man dies tut, so merkt man nämlich, daß die Arbeit von $\mathrm{C}_{\mathrm{AROL}}$ gar nicht in einem Forschungsprogramm liegt. Meine Frage wäre also: Wie können Sie die Forschungslogik von $\mathrm{C}_{\mathrm{AROL}}$ aufzeigen?

PH: Daß die hinreichende Abgrenzung vom organisierten Verbrechertum nicht gemacht ist, finde ich interessant, vielleicht wahr, aber irrelevant. Ich habe jetzt eben einiges gesagt über Wissenschaft, aber längst nicht alles. Ich habe lediglich gesagt, wie man Gegenstandsbereiche konstituiert, ich habe allerdings nicht gesagt, wie man dann, wenn man einen Gegenstandsbereich hat, so etwas wie eine Forschungslogik oder eine Methodologie, wie das auch genannt wird, begründet. In der Tat mag es so sein, daß man diese Ausführungen auch noch auf das organisierte Verbrechertum anwenden kann. Ich glaube, das liegt daran, daß Wissenschaft viel näher am Handwerklichen dran ist, als wir meinen. Wissenschaft ist wirklich aus dem Handwerk entstanden. Und ich habe den Eindruck, $\mathrm{da} ß$ wenn man genau diese Konstitutionsfragen untersucht, dann wird doch Wissen verwendet, ob's nun so handwerkliches Wissen ist oder ob's aus der medizinischen Praxis stammt - es wird halt etwas kontrollierter gemacht als man's sonst tut. So etwas Besonderes ist Wissenschaft gar nicht. Die Tatsache, daß in der theoretischen Physik z. B. der Gleichzeitigkeitsbegriff des Alltags umgestoßen worden ist, schreckt mich dabei gar nicht. Überall startet man mit dem Alltagswissen - in der Wissenschaft, im Handwerk -, und wenn man dann genauer hinsieht, muß man eben einiges davon revidieren.

PB: Die Kritik wäre jetzt natürlich, daß CAROL mit seinem degenerativen Forschungsprogramm in eine Sackgasse hineinführt.

PH: Carol hat hier überhaupt kein Forschungsprogramm vorgelegt. Was er gesagt hat, ist, wie man die 
grundsätzlichen theoretischen Fragen erörtert, die bei den Forschungsprogrammen schon vorausgesetzt werden. Das ist der Witz. Nur das hat er klargelegt.

Das ist ein etwas trauriger Zustand der Wissenschaftstheorie, daß sie entartet ist in eine Methodologie. Die Fragen, die die Wissenschaftstheorie stellt, sind keine wissenschaftsethischen Fragen mehr, auch keine erkenntnistheoretischen Fragen, sondern nur noch methodologische. In der Wissenschaftstheorie hat man ein bestimmtes Reflexionsdefizit, da man meint, $\mathrm{da} B$ es die Konstitutionsfragen nicht gäbe. CAROL hat sich die Mühe gemacht, an diese Konstitutionsfrage der Geographie heranzugehen. In vielen anderen Wissenschaften braucht man das nicht zu machen, da es so selbstverständlich funktioniert. Von diesem Selbstverständnis aus starten dann die Leute aus der analytischen Wissenschaftstheorie und vergessen diese Frage, zum Schaden der Geographie. $\mathrm{Da} B$ CARoL kein Forschungsprogramm gemacht hat, macht nichts. Der Begriff des Forschungsprogramms ist darauf nicht anwendbar. Was CAROL gemacht hat, geht einem Forschungsprogramm voraus. Ein Forschungsprogramm wäre es, wenn er sagen würde: «So, jetzt fangen wir einmal an, mit einer ganz einfachen Korrelation, und setzen die modellmäßig an, etwa die zwischen Lithosphäre und Athmosphäre und verfeinern sie, und dann nehmen wir noch die Anthroposphäre dazu und schauen, was mit dem $\mathrm{CO}_{2}$ passiert.» Aber so weit sind wir längst nicht. Wir befinden uns im Vorfeld davon.

PB: Aber $\mathrm{C}_{\mathrm{AROL}}$ wurde von den anderen Geographen so verstanden, daß er damit ein Forschungsprogramm vorgelegt habe.

PH: Das ist kein Argument. Dann ist das eben forschungspraktisch schiefgelaufen.

PB: Das würde dann heißen, daß die ganze Diskussion vorwissenschaftlich wäre.

PH: Das habe ich auch nicht behauptet. Was ich versucht habe, hier klarzumachen, ist etwas, das logisch vor der Definition von Forschungsprogrammen steht. Die Diskussion über Forschungsprogramme muß so etwas immer schon benutzen. Dies traue ich mir auch zu nachzuweisen, weil ich immer fragen kann: Wie begründet Ihr Eure Methoden in den Forschungsprogrammen? Bei dieser Frage kommen notwendigerweise auch die Eigenschaften der Objekte vor, dann frage ich: Woher wißt Ihr die? Dann komme ich genau auf die Konstitutionsfragen zurück. Man stellt diese Fragen vielfach nicht, weil es nicht nötig ist. Wenn der Molekularbiologe sagt: Ich interessiere mich für die biologisch relevanten Makromoleküle, weiß jeder, was gemeint ist. Aber wenn die Frage ist, was denn Landschaft sei, dann muß man sich das eben klarmachen. Dann muß die Geographie weiter zurückgehen in ihre erkenntnistheoretischen Fundamente.
TS: Ich glaube, daß $C_{A R O L}$ doch nicht viel gewonnen hat, wenn er sein Objekt so eingrenzt, daß es beliebig klein sein kann, bis zur molekularen Größe, aber auch beliebig groß, d.h. daß es die ganze Welt umfassen kann. Somit hat er sein Objekt als Gesamtheit definiert, welche sämtliche Wissenschaften umfassen kann. So kann er bei der Ausübung der Geographie seinem Objekt gar nicht mehr gerecht werden, da es allumfassend ist.

PH: Das sind jetzt, glaube ich, zwei Fragen. Die eine ist die, wie scharf die Auswahlkriterien sind, die ein jeweiliges Objekt begrenzen, und die andere Frage ist die, ob er sich mit dem Programm "überlupft» hat, weil es zuviele Korrelationen beinhaltet. Die erste Frage ist trivial. Das ist dasselbe, wie wenn man den Stein findet mit den Schriftzeichen. Da gibt es, weil wir viele verschiedene Interessen haben, viele verschiedene Möglichkeiten, den Stein zu untersuchen. Die andere Frage ist die, wie ambitiös dieser Ansatz ist. Der Ansatz ist in der Tat ambitiös. Genau so wie derjenige von den Molekularbiologen. Die Frage ist, ob sie jemals damit fertig werden. So ist Wissenschaft. Man sieht interessante Objekte, dann will man systematisch die Forschungsgegenstände benennen, dann sieht man, daß es viel Arbeit ist für die nächsten 200 Jahre - so auch in der Geographie. Die Physiker hatten, als sie anfingen, ein Forschungsprogramm mit der Planetenbewegung und mit der Gravitation. Das hat die Leute 200 Jahre lang in Atem gehalten. Das ist heute eine Sache, die man in den Anfängervorlesungen in theoretischer Physik im Prinzip in wenigen Stunden macht. Das wird in der Geographie nicht so einfach gehen, weil viele deskriptive Elemente drin enthalten sind. Da kann nicht die Vereinheitlichung der Theorien stattfinden wie in der Physik. Aber das macht überhaupt nichts. Dann haben die Kinder auch noch etwas zu arbeiten.

RN: Was mich an der Konstitution der Geographie von Hans Carol stört, ist, daß er kein eigentliches Problem stellt. Die Nationalökonomie geht dem Problem der Maximierung des Volkswohlstandes nach und die Medizin dem Wohlergehen des Menschen. Man kann fragen, wie sinnvoll diese Probleme sind, aber sie fordern einen heraus, sie interessieren. CAROL bietet unzählige Möglichkeiten, es gibt unzählige Möglichkeiten von Korrelationen, die man untersuchen kann. Was soll nun die Geographie an relevanten Problemen herausgreifen? Gerade die heutige Diskussion spiegelt gewisse Aspekte der kritischen Theorie, also einer gesellschaftskritischen Theorie, welche anstrebt, daß die Geographie etwas untersucht, das relevant ist in bezug auf die Probleme der Welt. Wie kann man nun eine Verbindung herstellen zwischen dem Ansatz von CAROL und einem gesellschaftskritischen Ansatz?

PH: Wer wird diese Frage dem Astronomen stellen? Menschen haben ein Interesse für die Natur. Ich 
glaube, wenn die Agrargeographen die Wahrheit gefunden haben, so ist auch etwas Interessantes für den Landwirt dabei. Aber zunächst einmal ist doch Wissenschaft eigentlich nicht so von vornherein der Praxis verpflichtet. Die Dinge, die wirklich relevant werden, das ist das Paradoxe, ergeben sich eben gerade daraus, daß man nicht dauernd auf Relevanz schielt. Das ist der Witz der Sache. Die großen und wirklich weitreichenden Dinge in der Wissenschaft ob man die gut findet oder nicht, ist eine andere Frage - sind gerade dadurch entstanden, daß die Leute nicht auf Relevanz geschielt haben. Wie bei HaHN und Strassmann mit der Atomspaltung. Die wollten das Atom ja gar nicht spalten, die wollten ja etwas ganz anderes machen, die wollten schauen, was für Transurane entstehen, wenn man Uran mit Neutronen bestrahlt. Dabei haben sie das Atom gespalten. Ob wir das nun wollen oder nicht, ist eine ganz andere Frage. Relevanz von Wissenschaft ist vielfach dort zustande gekommen, wo die Leute - und möglichst die besten Leute - frei haben spielen dürfen.

Dort sind historisch in der Wissenschaftsgeschichte die wichtigsten Sachen herausgekommen. Wenn ich nun das Maggiatal untersuche und dort die Korrelation zwischen Biosphäre und Boden und dazu noch die Anthroposphäre - z. B. wieviele Philosophen dort leben -, dann kann der Geograph das machen. Vielleicht interessiert es, vielleicht aber auch nicht, vielleicht wäre es besser, diese Untersuchung irgendwo zu machen, wo die Leute am Verhungern sind, daß man sich fragt, wie hängen Klima, Bewässerung und Boden zusammen.

CS: Die Methodologie, die Carol liefert, ist ja nicht die einzig mögliche Methodologie der Geographie.

PH: Das ist keine Methodologie, die uns CArol liefert. Es wird nicht gesagt, was die Methoden sind, sondern es wird das zugrundegelegt, von dem aus andere Leute dann sagen können, welche Methoden man sinnvoll anwenden kann.

CS: Gut, dann ist es eben eine Grundlegung der Geographie. Aber es ist sicher nicht die einzige Grundlegung der Geographie. Es gibt sicher andere, oder ich könnte mir mindestens andere vorstellen. Wenn verschiedene Grundlegungen da sind, muß man sich irgendwie einigen können. CAROL sagt eben, daß es die Grundlegung der Geographie sei, und das ist das Schlimme. Daher frage ich nun: Welches sind die Gründe, wieso wir diese Grundlegung überhaupt akzeptieren sollen? Carol selbst gibt keine Gründe an.

PH: Aber man kann doch nicht von jemandem verlangen, daß er sich gegen sämtliche möglichen Gegenargumente auch noch verteidigt. Da würde ich doch sagen: Ich bin Wissenschafter, ich bringe was. Dann kommt jemand mit einer anderen Grundlegung, und nun fangen wir an, miteinander zu reden. Der allgemeine Teil, den ich gebracht habe, hatte den
Sinn, daß man verschiedene Grundlegungen auf ihre Qualität hin vergleichen kann. Nämlich: Wie weit sind sie durchsichtig? Ich würde vermuten - dazu muß ich aber sagen, da $B$ ich kein Geograph bin -, daß die Grundlegung von $C_{A R O L}$ so weit ist, $\mathrm{da} B$ sie alles andere als Spezialfälle in sich aufsaugen kann. Das wäre meine Vermutung. Ich kann mir nichts vorstellen, was es sonst noch gibt, was Geographen sinnvoll untersuchen können, was damit nicht schon abgedeckt ist. Ich möchte mal ein Objekt wissen, das die Geographen untersuchen möchten, welches sich nicht in dieser Weise darstellen läßt. Wenn sie Philosophiegeschichte treiben möchten, dann möchte ich sagen, das sollen sie auch wirklich nicht tun. Philosophiegeschichte sollten schon die Philosophen machen. Ich mag die Geographen schon, aber auch nicht als theoretische Physiker, das ist ja auch ein Phänomen in der Anthroposphäre. Aber alles, was mir so bekannt ist an Geographie, läßt sich durchaus als Korrelation darstellen, auch wenn man den Stadtsoziologen dabei auf die Zehen treten will. Denn die Verbrecher benutzen immerhin auch die Straßen. Das ist dann eine Korrelation zur Lithosphäre. Das Programm ist sehr offen. Ich habe große Schwierigkeiten, mir irgendetwas vorzustellen, was dort nicht Platz hat. Das würde mich jedoch interessieren, da ich darüber zu wenig weiß.

HB: Das ist ein Punkt, welcher mich bei der Konstitution der Wissenschaft von CAROL stört. Die Konstitution ist so offen, daß mehr Platz hat, als nur die Geographie. Im Grunde genommen hat fast alles Platz...

PH: ... z. B. die Philosophie ist nicht drin...

HB: Wenn man dann noch betrachtet, was die Geographen in der Praxis tun, so kommt man darauf, $\mathrm{da} B$ sie eben alles tun. Es gibt Geographen, die machen rein nationalökonomische Studien...

PH: ... dann sind sie aber falsch am Platz ..., denn bei Carol wird gesagt, daß die Korrelationen das spezifisch Geographische seien. Wenn ich mich also nur in einer Sphäre bewege, z. B. in der Lithosphäre, dann komme ich mit den Geologen und den Felsmechanikern in Konflikt, weil die dort zuständig sind. Es gibt nicht nur für die einzelnen Sphären, sondern auch für einzelne Korrelationsarten bereits eine Wissenschaft. Die Meteorologen wissen auch, daß es einen Boden gibt. Dann sollen's eben die machen, welche das gelernt haben. Die Geographen müssen von der Geologie abschöpfen, damit sie über die Korrelationen sprechen können. Dann gibt es aber Konkurrenzen zu schon etablierten Wissenschaften, die sich bestimmte Korrelationen vorgenommen haben. Dann muß man sich halt einigen. Das ist ein ganz normaler Vorgang in der Wissenschaft. Das sind so Revierstreitigkeiten. Es wird einem offenen Programm etwas einverleibt, woran schon andere arbeiten. Dann muß man halt miteinander reden. 
EE: Ich habe mich über Ihren zweiten Teil gewundert Objektives Wissen stellen Sie dem bloß subjektiven Meinen gegenüber. Mir scheint, $\mathrm{da} ß$ «Meinen» nicht in den Hörsaal gehört. Das gehört auf die Straße. Vermuten ist jedoch nicht dasselbe. Dies gehört in den Hörsaal. Ich möchte versuchen, zwei andere Pole einander gegenüberzustellen. Dem objektiven Wissen stelle ich ein subjektives Wissen gegenüber. Sie haben zwar den subjektiven Blickwinkel später durch ein Hintertürchen mithereingeholt, aber ich möchte dafür plädieren, daß es ein subjektives $\mathrm{W}$ issen gibt. $\mathrm{Da} ß$ das objektive Wissen sehr stark vom Handwerklichen herkommt, haben Sie gesagt. Wir haben es dringend nötig, diesem objektiven handwerklichen Wissen, diesem quantifizierenden Wissen ein subjektives Wissen gegenüberzustellen. Es soll nicht nur der subjektive Blickwinkel, sondern, entschuldigen Sie jetzt, die subjektive Eingebung drin enthalten sein. Die Intuition ist ausschließlich subjektiv, aber unter Umständen ist sie die ganz große und zündende Anregung in diesem Wissen. Gerade in der Geographie haben wir das subjektive Wissen nötig. Statt "subjektiv» möchte ich nun "synthetisch» sagen und somit einem analytischen objektiven Wissen ein synthetisches subjektives Wissen gegenüberstellen. Im synthetischen Wissen spielt das Subjektive eine ganz große Rolle. Nun sind wir ganz nahe bei der Geographie, denn Geographie ist, so behaupte ich, im Prinzip ein synthetisches Wissen. Dann macht sie etwas, das niemand anders macht. Die Geographie hat nun den großen Vorzug, über das Handicap, daß wir soundso viel zusammenzutragen haben, daß wir eine totale Synthese vollbringen. Und diese totale Synthese, die sonst niemand macht, ist unser großes Glück. Ich möchte Ihnen empfehlen: Schreiben Sie doch lieber analytische Wissenschaft und synthetische Wissenschaft. Wenn Sie damit einverstanden sein könnten, wäre ich glücklich.

$\mathrm{PH}$ : Das ist nun eine ganze Menge, wozu ich etwas sagen muß. Das subjektive Meinen steht deshalb im Hörsaal, um es dem objektiven Wissen gegenüberzustellen. Um zu klären, was man mit einem Begriff meint, verfährt man häufig so, daß man einen oder mehrere Gegenbegriffe dagegensetzt. Dann wird einem plötzlich klar, was im Begriff steckt, da es vom anderen gerade ausgeschlossen ist. Dies war die Funktion.

Die Eingebung ist vom objektiven Wissen überhaupt nicht ausgeschlossen. Wenn Sie eine mathematische Aufgabe lösen oder einen Beweis finden müssen, dann finden Sie den selbstverständlich über die Eingebung, wie denn sonst? Aber die Objektivität von Wissenschaft zeigt sich in den Überprüfungsverfahren. Die Objektivität von Wissenschaft konstituiert sich nicht in der Weise, wie jemand drauf kommt, sondern in der Weise, wie es überprüft wird. Die Geschichte, ob der Herr KeKULÉ den Sechserring des Benzols geträumt hat oder ob's ihm sein kleiner Sohn zugeflüstert hat, ist insofern völlig uninteressant. Interessant ist, ob man nachher ein Experiment machen kann, um das nachzuprüfen. Mit objektivem Wissen ist überhaupt kein Gegensatz zur Intuition oder zur Eingebung gemeint.

Des weiteren würde ich sagen, wie immer man die Unterscheidung macht zwischen synthetisch und analytisch - all das, was Anspruch auf Wissen hat, fällt unter objektives Wissen. Daß CAROL die Geographie als eine Synthesewissenschaft auffaßt, ist die Konsequenz seines Ansatzes. Natürlich muß man die Elementarwissenschaften studieren, aber das spezifisch Geographische kommt da zustande, wo man die Korrelation der Sphären betrachtet.

HW: Du hast gesagt, daß Du uns als Geographen mit dem Ansatz von $C_{A R O L}$ beglückwünschst. Ich möchte bezweifeln, daß wir mit seinem Ansatz heute noch beglückt sind. Und zwar möchte ich die Fruchtbarkeit des Ansatzes aufgreifen. Ich behaupte, da $\beta$ es bis heute nicht gelungen ist, die Konstitution der Geographie nach Carol fruchtbar zu machen. Das Zentrale bei seiner Konstitution sind die Korrelationen. Du hast behauptet, daß diese Korrelationen unzweifelhaft seien. Genau dies möchte ich nun bestreiten. Das Beispiel, welches $\mathrm{C}_{\mathrm{ARO}} \mathrm{L}$ für eine Korrelation bringt, sind feuchte Luftmassen, die heranströmen und durch einen Berg zum Aufsteigen und damit zum Ausregnen gezwungen werden. Bis heute ist es nicht gelungen, die Korrelationen im Sinne Carols zu operationalisieren. Zudem ist es auch nicht gelungen, interessante Korrelationen zu finden, d.h. über triviale Beispiele hinauszukommen. Nachdem sich der Ansatz über 25 Jahre hinweg als unfruchtbar erwiesen hat, schlage ich vor, ihn aufzugeben.

PH: Worin besteht eigentlich die Fruchtbarkeit eines Ansatzes? Die Argumentationsstrategie wird sein, daß man die Fruchtbarkeit von Ansätzen immer vergleichen muß. Forschungsprogramme und deren Grundlegung müssen in Relation gesetzt werden. Ein Gesichtspunkt ist, wenn man's vergleicht mit den früheren Ansätzen, z. B. mit den Raumorganismen, dann bekommt man mit dem Ansatz von CAROL eine neue Fragestellung, die man davor nicht gehabt hat. Ich kann fragen, wie groß die Landschaft sein muß, wenn ich einen bestimmten Zweck verfolge. Das ist eine neue Forschungsfrage, wenn ich nicht mehr Landschaften als Raumindividuen ansehe. Ich muß mir nämlich die Frage stellen, wie groß ich die Landschaft bei einer bestimmten Fragestellung machen muß, z. B. bei der Frage, wie weiträumig klimatische Einflüsse sind. Diese Frage konnte man beim raumindividuellen Ansatz nicht stellen. Da bin ich aber Laie, das kann ich zu wenig beurteilen. Zur Fruchtbarkeit allgemein: Da würde ich gerne die Alternativen auf derselben Ebene sehen. Ich meine nicht auf der Ebene der schon aufgebauten Forschungsprogramme. Vielleicht ist es forschungspragmatisch ein Nachteil, daß CAROL nicht gleich bezüg- 
lich der Methodenbegründung dann auch noch das entsprechende Standardwerk geschrieben hat. Das ist aber ein Argument, das auf einer anderen Ebene liegt und hierfür nicht relevant ist. Ich möchte die anderen Forschungsprogramme, die ja eine solche Konstitutionsphase hinter sich haben, vergleichen bezüglich ihrer Konstitution des Gegenstandsbereiches mit der Konstitution von CAROL. Ich kann mir überhaupt nichts vorstellen, was hier nicht Platz hat und was sinnvoll von Geographen gemacht wird. Diejenigen, welche den Ansatz von CAROL kritisieren, sollen mir jetzt einmal eine Untersuchung sagen, die im Ansatz von CAROL nicht Platz hat.

TS: Das ist ja gerade das Problem, daß alles in diesem Ansatz Platz hat. Für den Geographen ist nichts gewonnen, wenn er diesen Ansatz zugrundelegt, weil er keine methodische Eingrenzung hat und weil sein Gegenstandsbereich immer noch unendlich gro $\beta$ ist. Deshalb muß er zunächst eine neue Eingrenzung vornehmen.

PH: Es ist einfach nicht wahr, daß alles Platz hat. Ich habe dies auch nie behauptet - sondern dementiert. Die Geographen sollten sich bitte heraushalten aus der theoretischen Physik, sollten sich bitte heraushalten aus der Philosophiegeschichte. Es hat nicht alles Platz, das ist schlicht nicht wahr.

TS: Aber es hat zu viel Platz.

PH: Aber nach welchem Maßstab sagst Du denn, daß zu viel Platz ist? Sollen denn die Molekularbiologen sagen, ja das Leben ist so vielfältig, daß wir uns nicht einmal mehr getrauen, Makromoleküle zu untersuchen? Das ist doch unsinnig. Natürlich ist es ein Riesengebiet, aber man braucht es ja auch nicht in einem Menschenleben abzuschließen. Kein vernünftiger Wissenschafter kann das hoffen. Die Forderung hat kein Augenmaß. Augenmaß haben für die Geographie heißt, nicht mehr zu verlangen, als in anderen vernünftigen etablierten Wissenschaften. Die Tatsache, daß hier keine Methodologie steht, hat den einfachen Grund, daß es eine Konstitution und keine Methodologie ist. Man darf der Konstitution nicht den Vorwurf machen, daß sie keine Methodologie ist. Man muß sagen: So jetzt geht's los, jetzt muß gearbeitet werden, jetzt bauen wir eine Methodologie auf, oder wir machen eine paradigmatische Pionierleistung im Sinne Thомas Kunns.

PB: Das ist genau, weshalb wir jetzt aneinander vorbeidiskutieren. Sie sagen, es sei die Grundlegung einer Methodologie. Aber CAROL hat das als Methode verstanden und Herr EgLI anscheinend auch. Das ist eben die synthetische...

PH: Aber die Stelle möchte ich sehen, wo Carol das sagt. Das ist schlicht nicht wahr. Wieso ist der Aufsatz denn in drei Abschnitte gegliedert? Der erste Abschnitt heißt: Welcher Art ist das Objekt geographischer Forschung? Darüber habe ich gesprochen. Der zweite Abschnitt heißt: Wie ist die geographische Wissenschaft ihrem Forschungsobjekt adäquat aufzubauen? Das sind einfach zwei verschiedene Fragen. Das war auch $C_{A R O L}$ ganz klar. Ich habe hier vor allem den Landschaftsbegriff behandelt. Und beim Landschaftsbegriff spielen die Methoden einfach noch nicht die Rolle, wie jetzt offensichtlich viele Leute meinen.

PB: Aber es werden dauernd methodische Bemerkungen gemacht. Formal ist es vielleicht nicht so, aber dem Inhalt nach. CARol sagt z. B.: «... die erschrekkende Konfusion selbst beim Grundbegriff der Geographie..." und: "Man ist versucht, vor dieser Wirrnis von Auffassungen zurückzuschrecken. Und doch müssen wir sie ordnen, Falsches ausrotten und auch den Mut haben, Neues zu prüfen.»

PH: Aber das ist doch keine Methodenfrage der Geographie, zu ordnen und Falsches auszurotten. Das darf man doch nicht als Methodologie hochstilisieren. Man muß sagen: Das ist der Sache nach doch keine Methodologie, und wenn er es einmal schreiben sollte, war das eine schwache Minute von ihm. Ein paar Sachen sind so, daß sie Mißverständnisse hervorrufen, aber die lassen wir weg, wir haben es jetzt gelernt. Es ist jetzt hoffentlich klar geworden, daß es um Konstitutionsfragen geht und daß wir hier nicht einen Sack voll Methoden bekommen haben.

HB: Gerade diese Konstitutionsfrage möchte ich eigentlich bezweifeln. Du gehst aus bei der Konstitution von einem Subjekt und einem Objekt. Ich meine, man müßte noch ein drittes Kriterium einführen, und zwar das Interesse.

PH: Das ist im Subjekt drin.

HB: Nein, das glaube ich nicht. Ich möchte es als selbständige Kategorie einführen.

PH: Wo gibt's denn das Interesse außer in den Subjekten?

HB: Interesse ist ja etwas Dazwischenliegendes.

PH: Also gut, wir brauchen ja hier nicht terminologisch zu streiten. Interessen sind etwas, was ich jetzt bei den Subjekten verorte. Wenn Du die Interessen herausnimmst, dann wird Wissen oder Erkenntnis eine dreistellige Relation, ist mir auch egal. Wenn wir dann nur dasselbe meinen.

HB: Ich möchte mit dem Beispiel vom Stein antworten. Es ist eben nicht dasselbe, ob ich den Stein mit dem Interesse des Kristallographen oder mit demjenigen des Linguisten betrachte. Als Linguist interessieren mich die Zeichen und nicht der Stein, und dies ist nicht dasselbe.

PH: Das ist richtig. Daher habe ich auch zwischen dem Stein als materiellem Ding und als Objekt einer Wissenschaft unterschieden. In der Physik ist das altbekannt. Man unterscheidet physikalische Systeme 
von thermodynamischen. Thermodynamische sind solche, wo ich bereits die Begriffe, mit denen ich das System beschreiben will, spezifiziert habe.

HB: Aber es gibt viele Wissenschaften, die sich mit demselben Objektbereich, aber mit einer speziellen Perspektive befassen.

PH: Jetzt müssen wir aber genauer reden: Die Wissenschaften beschäftigen sich mit den gleichen Dingen, die in verschiedene Objektbereiche fallen.

HB: Das Objekt von Carol ist die Landschaft. Aber es gibt verschiedene Wissenschaften, die an der Landschaft interessiert sind, jedoch mit ganz verschiedener Perspektive, und das Interesse entscheidet dann schlußendlich.

PH: Das Letzte ist nicht mehr richtig, alles andere ist richtig. Das Spezifische an den Landschaften ist das Korreliertsein der Sphären. Und nun sind wir wieder am selben Punkt wie vorher. Selbstverständlich gerät die Geographie in Konkurrenz zu anderen Wissenschaften. Aber das Interesse bestimmt nicht die Methode in letzter Instanz. Sondern es ist immer so, daß es dieses Zusammengreifen ist, von Objekt als auch Subjekt - und wenn Du noch willst - auch von Interesse.

ER: Nach Deinen Ausführungen frage ich mich schlußendlich, warum die Geographie dann in einer Dauerkrise steckt. Vom Subjekt her frage ich mich, ob wir nicht der Gesellschaft gegenüber eine gewisse Verantwortung tragen und nur Dinge untersuchen sollten, die etwas bringen.

PH: Woher diese Dauerkrise kommt, weiß ich nicht. Ich habe bereits gesagt, daß das Schielen nach Relevanz so eine Geschichte ist. Wenn jemand als Geograph etwas unmittelbar Wichtiges machen will, dann kann ich mir vorstellen, daß er die Sahelzone untersucht. Aber das ist wieder eine Frage des Augenmaßes. Wenn wir nur noch schauen würden, $\mathrm{da}$ wir etwas zu essen haben, uns also nichts anderes mehr interessiert, keine Kunst, keine Kultur, keine Astronomie mehr, ich weiß nicht. Ich finde beide Extreme falsch, also nur noch auf Relevanz achten oder gar nicht mehr. Aber wo der Weg in der Mitte ist, weiß ich nicht. Wissenschaftshistorisch würde ich jedoch im Auge behalten, daß die Leute auf die Möglichkeit der Linderung von sozialen Mißständen gekommen sind, als sie nicht auf Relevanz schielten. Diese paradoxe Struktur sollte man im Auge behalten.

OW: Ich kannte HaNS CAROL persönlich gut; wir diskutierten tagelang über diese Fragen. Und wir haben immer wieder gesagt: Jetzt muß es dann losgehen mit der konkreten methodologischen Arbeit. CARol war nie der Meinung, etwas Endgültiges zuwege gebracht zu haben. Im Gegenteil, er verglich seine Leistung gerne mit dem Aushub für ein Haus, aber nie mit dem schon gebauten Haus. Noch von
Kanada aus hat er später bedauert, nichts Fortgeschritteneres vorlegen zu können. Ich verstehe Ihre Kritik an CARols allgemeinen, schwer operationalisierbaren Vorstellungen, und ich glaube, da $B$ auch er ihr beipflichten würde. Trotzdem, scheint mir, müssen wir für seine Aktivität auf theoretischem Gebiet dankbar sein und sie im übrigen auch ein bißchen in ihrer Situationsbedingtheit sehen. Damals wurde nämlich wenig über Fragen der geographischen Theorie gesprochen, und es war nicht immer möglich, eigene Ideen an Gegenpositionen zu messen. Man kam sich darum zeitweise als Einzelgänger vor. Anstatt allgemeinere klärende Diskussionen gab es eher einfach Zustimmung oder Ablehnung. Vor allem die subjektbezogenen, standpunktbedingten Betrachtungssysteme stießen, wohl wegen eines Mißverständnisses, da und dort - z. B. auch bei Vertretern der Fakultät - auf Ablehnung. Ich bin jetzt angenehm überrascht, daß im Vortrag eines Vertreters der Theoretischen Physik eine diesen Problemen gegenüber positive Haltung sichtbar wird.

PH: Ich fürchte, daß sich das Klima dort nicht so geändert hat. Wenn ich das so sage, dann weniger als theoretischer Physiker, sondern mehr als Philosoph. Bei den Philosophen wird seit 199 Jahren so geredet. 1781 kam die "Kritik der reinen Vernunft" heraus. Dort ist die kopernikanische Wende. KANT bemerkte, daß das Zustandekommen von Wissen wesentlich durch Eigenschaften des Subjekts bestimmt ist. Die Behauptung als Philosoph ist die, daß das Subjektive immer mitkonstitutiv ist für das Wissen. Die Erklärung, wieso Naturwissenschafter dies von sich weisen, ist die, daß die Naturwissenschafter im allgemeinen sich das nicht bewußt zu machen brauchen. Ich kann Ihnen ein sehr extremes Beispiel geben, woran man so etwas merkt: Wenn jemand aus dem 20. Stockwerk springt, kann man als Physiker in der Tat daran das Fallgesetz verifizieren, aber Sie merken, daß es da ganz unangenehm wird. Als Physiker muß man sich eben nicht bewußt machen, daß eine Auswahl von Eigenschaften als die "physikalischen» Eigenschaften eine willkürliche Auswahl ist, d.h. eine subjektbestimmte. Man merkt eben, daß die Auswahl, dies so zu betrachten, sehr willkürlich, d. h. subjektiv ist. Der zweite Punkt ist der, daß eben die Meinung besteht, daß objektives Wissen ganz vom Objekt her bestimmt ist. Das ist eine alte empiristische Tradition. Das Subjekt muß sich einfach auslöschen. Alles, was das Subjekt dazubringt in diese Wissensrelation, ist schlecht und stört die Reinheit der Darstellung. Das ist die Tradition, in welcher die Naturwissenschafter stehen, und daher hat für sie das Subjekt einen so negativen Klang.

TK: Es ist ja nicht sinnvoll, eine Wissenschaft der zwei Meter langen Dinge zu machen. Ich frage mich nun, ob die Korrelationen ein sinnvolles Objekt für eine Wissenschaft sind. Diese Korrelationen sind so unterschiedlich, daß ich darauf keine Wissenschaft 
aufbauen kann. Dann kommt das Problem, daß der Geograph, wenn er an konkrete Problemstellungen herankommt, immer schon eine andere Wissenschaft vorfindet.

PH: Das kann man nun sehr sinnvoll diskutieren. Wie weit dies nun pragmatisch sinnvoll ist, daß die Geographie ein so großes Wissensziel setzt, darüber kann man sich streiten. Zweierlei kann man dazu sagen. Erstens sollte man sich von großen Wissenszielen nicht entmutigen lassen. Wie ich schon vorher gesagt habe, haben das andere Wissenschaften auch. Zweitens ist die große Unterschiedlichkeit der Korrelationen sicher ein forschungspraktisches Problem, aber kein grundsätzliches. Ich denke da an die Physik: Da gibt es etwa seit 100 Jahren die Unterscheidung von experimenteller und theoretischer Physik. Die Apparate und die Theorien wurden immer komplizierter, und dann gab es diese sich auch institutionell niederschlagende Arbeitsteilung. Und die Zusammenarbeit ist nicht immer ganz einfach. Analoges kann ich mir für die Geographie vorstellen, ohne daß dabei die Einheit der Geographie aufgegeben wird. Da kann es dann durchaus eine Arbeitsteilung zwischen Anthropogeographen und Naturgeographie geben, wie das auch bei CAROL gesagt wird.

KI: Theo Keller, ich glaube, Du hast Dein Unbehagen über die Synthesefähigkeit des Geographen zum Ausdruck gebracht. Dieses Unbehagen ist uns allen gemein, also daß wir Angst haben vor diesem etwas zu großen Ziel.
MEUSBURGER Peter: Beiträge zur Geographie des Bildungs- und Qualifikationswesens. Innsbrucker Geographische Studien, Band 7, Selbstverlag für Geographie der Universität Innsbruck, Textband 231 S., Kartenband mit 37 Karten, 1980, S 375.--.

Meusburger stellt in diesem Werk eine Anzahl verschiedener Fragestellungen und Untersuchungsergebnisse vor, in welchen die räumliche Differenzierung des Ausbildungsniveaus der Bevö1kerung entweder eine erklärende oder eine zu erklärende Variable darstellt.

Die behandelten Themen reichen von einer Beschreibung und Erklärung des regionalen Verbreitungsmusters der Arbeitsplätze nach ihren Ausbildungsanforderungen (Kap. 2), über die bildungsmässige Differenzierung der Wohnbevö1kerung zwischen Grossstadtquartieren (Kap. 3), das Ausbildungsniveau der ethnischen und religiösen Minderheiten in Oesterreich (Kap. 4), das Bildungsverhalten der Kinder in Abhängigkeit der sozialen Schichtzugehörigkeit der Eltern und der regionalen Herkunft (Kap. 5 und 6) bis zum Einfluss des Ausbildungsniveaus auf die räumliche Mobilität (Kap. 7). Jeder Themenkomplex wird durch umfangreiches, statistisches Datenmaterial für das Fallbeispiel Oesterreich illustriert.

Diese sehr breit angelegte Thematik geschieht bewusst und in der Absicht "den Nachweis zu erbringen, dass der ausbildungs- und qualifikationsorientierte Ansatz... bei vielen traditionellen und modernen Richtungen und Fragestellungen in der Anthropogeographie wissenschaftlich sehr ergiebig sein kann..." (S. 16). Schwerpunkt dieses Werkes und gleichzeitig wissenschaftlich ergiebigster Teil bildet die Analyse des regionalen Verbreitungsmusters der Arbeitsplätze nach dem Ausbildungs- und Qualifikationsniveau. Empirisch gut belegt und in umfangreichem Kartenmaterial dargestellt, weist Meusburger vorerst folgende Gesetzmässigkeiten dieses Verbreitungsmusters nach: Je höher die für einen Arbeitsplatz benötigte Ausbildung ist, desto grösser die räumliche Konzentration dieser Arbeitsplätze auf einige wenige Zentren. Demgegenüber ist die grösste regionale Gleichverteilung bei den Arbeitsplätzen mit den niedrigsten Ausbildungsanforderungen festzustellen. In einem weiteren Schritt präsentiert der Autor sodann einen sehr anregenden theoretischen Erklärungsansatz für diese ungleichmässige räumliche Verteilung aus dem Bereiche der Organisations-, Kommunikations- und Systemtheorie. Er stellt dar, dass mit fortschreitender Arbeitsteilung und zunehmender Grösse und Komplexität von wirtschaftlichen und sozialen Organisationen auch die Zahl der hierarchischen Kontroll- und Steuerungsinstanzen zunehmen musste und dass Planungs-, Koordinations- und Entscheidungsfunktionen im Zuge dieser Optimierung von Organisations- und Führungsstrukturen auf immer höhere Ebenen der Organisationshierarchie verlagert worden sind. Aufgrund unterschiedlicher Anforderungen der einzelnen Ebenen an das Komounikationspotential eines Standortes führten diese Optimierungsprozesse gemäss diesem theoretischen Erklärungsansatz gleichzeitig zur Entstehung einer Hierarchie der Standorte bzw. zur erwähnten Konzentration von hochqualifizierten beruflichen Tätigkeiten und Führungspositionen auf wenige Zentren.

Regionale Unterschiede in der Qualifikationsstruktur der Arbeitsplätze stellen eine wesentliche Ursache für die heute in praktisch allen Ländern festzustellenden regionalen Wohlstandsunterschiede dar, die in zunehmendem Masse als wirtschaftlich und gesellschaftspolitisch unerwünscht erachtet werden. Immer mehr Länder verfügen deshalb über ein politisches Instrumentarium zum Abbau dieser räumlichen Ungleichgewichte. Meusburgers theoretische und empirische Arbeit zur regionalen Qualifikationsstruktur der Arbeitsplätze stellt somit auch einen wertvollen Beitrag an eine problemorientierte Anthropogeographie dar.

GH 1/82 Elisabeth Bühler-Conrad, Zürich 